\title{
What's new in acne and rosacea?
}

\author{
Jonette Keri, $\mathrm{MD}, \mathrm{PhD}^{1,2}$
}

\section{- Abstract}

Acne and rosacea are common conditions seen every day by dermatologists. This review will discuss the most recent therapeutic options for patients with these conditions. Specifically, for acne, there will be a discussion of the use of isotretinoin at higher cumulative doses as well as a new formulation of isotretinoin, isotretinoin-lidose. Adult women with acne represent a growing population of patients who present for treatment of acne; the use of hormonal therapies as well as topical dapsone gel will be reviewed for these patients. For rosacea patients, the new topical agents - brimonidine gel and ivermectin cream - will be reviewed, with a discussion on possible rebound phenomenon from brimonidine. Finally, future treatments in the pipeline will be discussed.

Semin Cutan Med Surg 35:103-106 @ 2016 Frontline Medical Communications

\section{Acne}

Acne is a common and complex skin disease of the pilosebaceous unit. It is clinically characterized by open and closed comedones, papules, pustules, and nodules affecting the face, neck, chest, back, and upper extremities. Although it is a common disease of teenagers, it can affect the pediatric and adult population as well; and in moderate-to-severe cases, acne can cause significant scarring for the patient. The pathophysiology of this condition is complex; the newer developments involve inflammation directed by the innate immune system, and there are new findings elucidating the role of hyperkeratinization, sebum production, and Propionibacterium acnes. ${ }^{1}$ These advances will help target more pathways for treatment in the future.

\section{Treatments}

\section{Isotretinoin dosing and formulations}

Isotretinoin-lidose. A new formulation of isotretinoin - isotretinoin-lidose - was recently introduced to the market with the goal to reduce the bioavailability differences of isotretinoin during fed and fasted states compared to standard isotretinoin. This medication has the advantage of providing good levels of absorption even when patients are in a fasting state. Both medications -

\footnotetext{
${ }^{1}$ Associate Professor of Dermatology and Cutaneous Surgery, University of Miami, Miller School of Medicine, Florida.

${ }^{2}$ Chief, Dermatology Service, Miami VA Hospital, Florida.

Disclosures: Dr Keri is a consultant for Roche Pharmaceuticals. Correspondence: Jonette Keri, MD, PhD; Department of Dermatology and Cutaneous Surgery; University of Miami, Miller School of Medicine; 1600 NW 10th Avenue, RMSB 2023A; Miami, FL 33136. Email: jkeri@med.miami.edu.
}

isotretinoin-lidose and isotretinoin - were bioequivalent during a fed state during an open-label, single-dose, 4-treatment crossover study. ${ }^{2}$ Specifically, mean plasma levels of the isotretinoin-lidose formulation during fasting reached $66.8 \%$ of that observed with a fatty meal; and those of the standard isotretinoin formulation only reached $39.6 \%$ of that observed with a fatty meal. ${ }^{2}$ An additional study revealed no difference in safety or efficacy with this newer formulation versus generic isotretinoin. ${ }^{3}$ Thus, this new formulation of isotretinoin represents an option for patients who are unable to take the medication with food, specifically a high-fat meal, and may improve clinical outcomes.

Cumulative dosing. There have been various dosing regimens for isotretinoin over the years ranging from low-dose, long-term protocols to very high, cumulative-dose practices The most evidence lies with using cumulative dosing for appropriate patients. This cumulative dosing range has traditionally been between 120 $150 \mathrm{mg} / \mathrm{kg}$. However, recent studies suggest the risk of relapse may decrease when a higher cumulative dose is used. An early retrospective study showed a decrease in relapse rates. ${ }^{4} \mathrm{~A}$ follow-up, prospective study using a higher cumulative dose of isotretinoin added confirmation to this concept. In this study, the patients were divided into 2 groups based on $\geq 220 \mathrm{mg} / \mathrm{kg}$ vs $<220 \mathrm{mg} / \mathrm{kg}$. At one year after completion of isotretinoin, the authors found patients in the higher group $(220 \mathrm{mg} / \mathrm{kg}$ or more $)$ had a significantly decreased relapse risk. ${ }^{5}$ Rash was the only adverse event significantly more common in the high-dose group,,$^{5}$ lending support for the use of a higher cumulative dose.

Topical dapsone. Topical dapsone $5 \%$ gel is a newer member of the armamentarium of topical acne treatments since its approval in 2008. Dapsone gel has found a specific indication in the treatment of adult female acne. Specifically, topical dapsone gel appears to be more efficacious in adult females than in adolescent females, although it helps patients in both age groups. Significantly greater percentage reductions in both noninflammatory $(P<.0001)$ and total lesion counts $(P=.0008)$ were observed in the adult group as compared to the adolescent group. ${ }^{6}$

Topical dapsone represents a novel type of treatment for acne. It was shown to be safe and efficacious over a one-year period; ${ }^{7}$ and then expanded to include safety in patients with glucose-6-phosphate dehydrogenase deficiency (G-6-PD deficiency). ${ }^{8}$ However, in postmarketing surveillance, rare cases of methemoglobinemia were identified. The US Food and Drug Administration (FDA) added safety labeling changes in July 2015. These warnings reported are summarized below: ${ }^{9}$

"Cases of methemoglobinemia, with resultant hospitalization, have been reported post marketing in association with dapsone $5 \%$ gel treatment. Patients with G-6-PD, congenital or idiopathic methemoglobinemia are more susceptible to drug-induced methemoglobinemia and should avoid use of this product. Signs and 
symptoms of methemoglobinemia may be delayed some hours after exposure. Initial signs and symptoms of methemoglobinemia are characterized by a slate grey cyanosis seen in buccal mucous membranes, lips and nail beds. Patients are advised to stop dapsone gel and seek medical attention in the event of cyanosis. Dapsone can cause elevated methemoglobin levels particularly in conjunction with methemoglobin-inducing agents and prescribers should review medications that can induce methemoglobinemia. The above warning represents a response to a rare but serious adverse event, discovered after the medication was on the market, and is best addressed with simple patient evaluation and education.

"It is always prudent to note that topical application of dapsone gel followed by benzoyl peroxide can result in a temporary local yellow to orange discoloration of the skin and facial hair which was temporary and resolved within days to about 2 months at the longest." 9

Very recently, dapsone $7.5 \%$ gel has been approved for the treatment of acne in patients 12 years and older. In the double-blind, 12 -week study, both inflammatory and noninflammatory acne lesion counts decreased. Using dapsone $7.5 \%$ gel once a day for 12 weeks demonstrated a $>50 \%$ reduction in inflammatory lesions and noninflammatory lesion counts decreased by $>40 \%$ at 12 weeks. Side effects were minimal with application site dryness occurring in $1.1 \%$ of patients and pruritus in $0.9 \%$. The warnings remain the same for this higher-concentration product. ${ }^{10,11}$

\section{Hormonal therapies}

Antibiotic stewardship remains a goal for physicians treating the chronic conditions of acne and rosacea. Specifically for acne, the use of oral contraceptives as well as the antiandrogen spironolactone represents ways to use fewer antibiotics, both orally and topically, in acne patients. A meta-analysis by the Cochrane guidelines reviewed randomized controlled trials comparing the efficacy of antibiotics and oral contraceptive pills (OCP) in managing acne. The conclusions are that while antibiotics may be superior at 3 months, OCPs are equivalent at 6 months in reducing acne lesions and may represent an alternative to antibiotics for long-term acne treatment. $^{12}$

Although spironolactone is an older medication, its use has recently increased in the treatment of acne. In the past, the practice of having patients get routine blood tests for potassium - given the risk of hyperkalemia - has deterred some from prescribing this medication. A recent retrospective review in young, healthy women taking this medicine found no increased risk of hyperkalemia versus the general population. ${ }^{13}$ Specifically, there were only $13 / 1,802$ abnormal potassium values; of the 13 abnormal potassium values, 6 had normal values when repeated and the remaining 7 had no action taken because of this result. ${ }^{13}$ However, patients on other medications that can increase potassium or who eat high amounts of potassium-containing foods should be educated and monitored.

\section{Diet and alternative treatments}

A Cochrane review of diet and complimentary medicines found that there was some evidence for a low glycemic load (LGL) diet in treating acne as well as tea tree oil and bee venom. The authors concluded there was low-quality evidence from single trials to support this conclusion. Their review did not support evidence for other herbal or complimentary medicines. ${ }^{14}$

\section{Lasers and lights}

Photodynamic therapy continues to be used for acne. A review of the literature suggests that photodynamic therapy is safe with acceptable adverse events and can be used in a variety of ways with different photosensitizers including aminolevulinic acid (ALA), methylaminolevulinate (MAL) and indole-3-acetic acid (IAA) as well as different light sources, red light, pulsed-dye laser (PDL), intense-pulsed light (IPL), long-pulsed dye laser (LPDL), and green light. Of these, ALA works best when combined with red light in the treatment of acne. ${ }^{15}$ Laser therapy is advancing to include more treatments for active acne; whereas in the past, the focus for many had been on acne scarring. Laser treatments with long-term antiacne therapeutic effects - including a decrease in oiliness were recently noted following use of the $1,550 \mathrm{~nm}$ erbium:glass (Er:glass) fractional laser; some patients were followed for 2 years and continued to have benefit. ${ }^{16}$ The use of topically applied gold nanoparticles combined with a laser application is also being investigated for treatment of acne. When exposed to laser light at 800 $\mathrm{nm}$, gold nanoparticles selectively absorb light and subsequently produce heat. After topical application, these light-absorbing nanoparticles penetrate down the pilosebaceous unit with the assistance of a massage tool. Subsequent laser application to the skin causes light absorption by the gold nanoparticles, generation of heat, and selective destruction of the pilosebaceous unit. Results of a study showed a significant reduction in inflammatory lesions when topically applied gold nanoparticles were followed by the application of a $800 \mathrm{~nm}$ diode laser utilizing 3 treatments at oneweek intervals. Further studies are needed; but initial results are encouraging for this new treatment approach. ${ }^{17}$

Hand-held home devices as well as in-office vacuum-assisted laser and light devices are available and represent alternatives for patients looking for nontraditional therapies.

\section{Future acne therapies}

Medications in development for the treatment of acne include cortexolone $17 \alpha$-propionate, a nitric oxide-releasing nanoparticle and minocycline foam. ${ }^{18}$ Cortexolone $17 \alpha$-propionate is a topical antiandrogen; in a pilot study with male subjects, it was shown to decrease acne lesions over placebo and tretinoin $0.05 \%$ cream. ${ }^{19}$ This compound also had better tolerability than tretinoin in the study; however, systemic absorption and use in women of child-bearing years have yet to be defined. Another interesting therapeutic modality is a nitric oxide-releasing nanoparticle (NO-np) recently studied in vitro for its antimicrobial as well as immunomodulatory effects. These particles were shown to inhibit the $p$ acnes-stimulated inflammation cascade participants with minimal toxicity to keratinocytes. ${ }^{20}$ Minocycline $4 \%$ foam is also moving toward the market for the treatment of acne. Noted in the literature as a future treatment for impetigo, ${ }^{21}$ clinical trials for acne show a reduction in inflammatory $(71 \%)$ and noninflammatory $(73 \%)$ lesions at 12 weeks. ${ }^{22}$

Sebum reducers are also an area of research. In addition to topical androgens, acetylcholine inhibitors and peroxisome proliferator-activated receptor (PPAR) modulators may be promising for the control of sebum. ${ }^{23}$ Pilot studies with melanocortin receptor antagonists have shown decreased sebum with good tolerability. ${ }^{24}$ 


\section{Rosacea}

Rosacea is a common facial dermatosis of an inflammatory nature. The condition is characterized by 4 types: 1) erythromatotelangiectatic; 2) papulopustular; 3) phymatous, and 4) ocular. ${ }^{25}$ More recently, the 2 most-common clinical presentations for rosacea have been described as: 1) diffuse centrofacial erythema without papulopustular lesions; and 2) diffuse centrofacial erythema with papulopustular lesions. ${ }^{26}$ Although known to many dermatologists, characterization of skin as rosacea-prone skin is an important factor for patient education and treatment.

Rosacea is seen in all skin types, but a preponderance is seen in patients with fairer skin. It is seen in both genders with women tending to have symptoms at an earlier age and men having more phymatous changes. The onset is typically the 3rd or 4th decade of life and the condition is often associated with flares followed by more quiescent periods.

The primary physical findings include erythema of the facial skin, flushing, sensitivity of the skin, papules, pustules, telangiectasias, and phymatous changes of the face. Most patients experience a slow progression of the disease over the years.

The pathophysiology of rosacea is complex and not yet fully understood. However, evidence suggests that disruption of the epidermal barrier, triggering of the innate immune system, and neurovascular dysregulation all contribute to the disease. ${ }^{27}$ Clinically, there is association between rosacea and sensitive skin. This translates into a lower threshold for irritation which in turn is associated with higher transepidermal water loss (TEWL) and less stratum corneum hydration. The patient with such skin is then more susceptible to the known triggers of rosacea, such as heat, ultraviolet light, spicy food, alcohol.

\section{Treatment}

Conventional treatments for rosacea include antibiotics of the tetracycline class; and when those are contraindicated, the macrolides or penicillin/synthetic penicillin class have been used. Topically, the mainstays of treatment include metronidazole, azelaic acid, sodium sulfacetamide and sulfur. Additionally, the 2 most recent advances in topical treatment for rosacea include topical brimonidine and topical ivermectin. A review of the literature for efficacy by Cochrane methodology suggests there is high-quality evidence for topical brimonidine, azelaic acid, ivermectin, oral doxycycline, and isotretinoin. There is lower-quality evidence for topical metronidazole, oral tetracycline, laser- and light-based therapies, and topical cyclosporine for ocular rosacea. ${ }^{28}$

\section{Brimonidine}

Brimonidine is a selective $\alpha 2$-adrenergic receptor agonist. This medication was first approved as an ophthalmic solution for the treatment of open-angle glaucoma. As a treatment for rosacea, it induces vasoconstriction of the superficial small $(<200 \mathrm{~nm})$ vessels of the face. Brimonidine also has some anti-inflammatory effects shown in mouse-ear models where edema is lessened with its use. ${ }^{29}$ A long-term safety study with brimonidine $0.33 \%$ gel with a 12-month follow up found that the adverse events were higher at the beginning but lessened over time. The most common adverse events during the 4th quarter of the year-long study were flushing, worsening of erythema, worsening of rosacea, contact dermatitis, and pruritus. In general, skin side effects were mild to moderate. ${ }^{30}$ Due to the mechanism of action of this medication as a potent $\alpha 2$-adrenergic receptor agonist, theoretical risks include lowering blood pressure. The warnings/precautions listed by the FDA for this medication state brimonidine gel should be used with caution in patients with a variety of medical conditions including patients with severe, unstable, or uncontrolled cardiovascular disease. ${ }^{31}$ Serious adverse reactions following accidental ingestion of brimonidine gel by children have been reported. ${ }^{31}$ Although these precautions are mainly theoretical, simple patient evaluation and education can guard against rare adverse events.

The question of rebound erythema with brimonidine gel has been investigated after initial case reports showed a noticeable increase in erythema after use of the medication in a few patients..$^{32,33}$ What followed was further delineation of the erythema and the timing of the redness. The term "rebound" is often misinterpreted. A consensus group put forth the idea of better defining this term for patients after finding that about $10 \%-20 \%$ of real-world patients were getting some type of erythema. ${ }^{34}$ Their proposed new definitions of the types of erythema after brimonidine were classified into 4 groups: 1) paradoxical erythema that comes 3-6 hours after application and the redness can be worse; 2) return to baseline erythema after 10-12 hours; 3 ) exaggerated recurrence of erythema redness that is greater than baseline that appears 10-12 hours after therapy wears off; 4) allergic contact dermatitis - redness usually accompanied by other signs such as pruritus that typically occurs after 3-4 months of therapy. ${ }^{33}$ Most importantly, what came from this consensus is a strategy to educate patients about their condition of rosacea, to educate them about the use of this medication, and as to how to treat subsequent erythema.

\section{Ivermectin}

Ivermectin cream represents a new treatment for papulopustular rosacea. Initial clinical trials show a greater proportion of subjects in the ivermectin-treated group achieved a "clear" or "almost clear" response, $38.4 \%$ and $40.1 \%$ versus $11.6 \%$ and $18.8 \%$ for vehicle (both, $P<.001$ ), respectively. ${ }^{35}$ Although the mechanism of action is unknown, ivermectin has anti-inflammatory and antiparasitic activities. It represents a safe and well-tolerated option for treatment. In a head-to-head study between metronidazole cream $0.75 \%$ and ivermectin cream $1 \%$, both creams improved the papulopustular rosacea, but the ivermectin cream was found significantly superior in terms of reduction from baseline in inflammatory lesions. ${ }^{36}$

\section{Ocular rosacea}

Ocular rosacea is sometimes missed in patients in the dermatology clinic. As a reminder, oral antibiotics for rosacea will help this condition and a recent study supported use of low-dose doxycycline for ocular rosacea. In this study, the patients received $40 \mathrm{mg}$ of doxycycline for 12 weeks and had significant improvement in their ocular symptoms. ${ }^{36}$ Additionally, even after discontinuation of the doxycycline, some patients had continued improvement for 17 months. ${ }^{37}$ Finally, in a 3-month study comparing artificial tears to cyclosporine eye drops used twice a day, cyclosporine $0.03 \%$ was more effective in treating lid and corneal changes associated with ocular rosacea. ${ }^{38}$

\section{Conclusion}

Acne and rosacea represent conditions that dermatologists see on a 


\section{TABLE. What's new in acne and rosasea}

Acne
- Increased dosing with isotretinoin
- New formulation of isotretinoin
- Oral contraceptives are as effective as oral antibiotics at 6 months
of use
- No need to check potassium levels in young, healthy women being
treated for acne
- Topical dapsone gel has a role in treating adult female acne
patients
Rosacea
- Topical brimonidine for erythema
- Watch for erythema in 10\%-20\% of patients using topical
brimonidine and educate patients
- Topical ivermectin for rosacea

daily basis. Reviewing the latest advances in the treatment of these illnesses will help both patient and practitioner. The Table presented provides a quick reference for what is new in acne and rosacea.

\section{References}

1. Das S, Reynolds RV. Recent advances in acne pathogenesis: implications for therapy. Am J Clin Dermatol. 2014;15(6):479-488

2. Webster GF, Leyden JJ, Gross JA. Results of a Phase III, double-blind, randomized, parallel-group, non-inferiority study evaluating the safety and efficacy of isotretinoin-Lidose in patients with severe recalcitrant nodular acne. J Am Acad Dermatol. 2014;13(6):665-670.

3. Webster GF, Leyden JJ, Gross JA. Comparative pharmacokinetic profiles of a novel isotretinoin formulation (isotretinoin-Lidose) and the innovator isotretinoin formulation: a randomized, 4-treatment, crossover study. J Drugs Dermatol. 2013;69(5):762-767.

4. Coloe J, Du H, Morrell DS. Could higher doses of isotretinoin reduce the frequency of treatment failure in patients with acne? J Am Acad Dermatol. 2011;65(2):422423.

5. Blasiak RC, Stamey CR, Burkhart CN, Lugo-Somolinos A, Morrell DS High-dose isotretinoin treatment and the rate of retrial, relapse, and adverse effects in patients with acne vulgaris. JAMA Dermatol. 2013;149(12):1392-1398.

6. Del Rosso JQ, Kircik L, Gallagher CJ. Comparative efficacy and tolerability of dapsone 5\% gel in adult versus adolescent females with acne vulgaris. $J$ Clin Aesthet Dermatol. 2015;8(1):31-37.

7. Lucky AW, Maloney JM, Roberts J, et al; Dapsone Gel Long-Term Safety Study Group. Dapsone gel 5\% for the treatment of acne vulgaris: safety and efficacy of long-term (1 year) treatment. J Drugs Dermatol. 2007;6(10):981-987.

8. Piette WW, Taylor S, Pariser D, Jarratt M, Sheth P, Wilson D. Hematologic safety of dapsone gel, 5\%, for topical treatment of acne vulgaris. Arch Dermatol. 2008;144(12):1564-1570.

9. US Food and Drug Administration. Aczone (dapsone topical gel) 5 Percent Safety Labeling Changes Approved By FDA Center for Drug Evaluation and Research. http://www.fda.gov/Safety/MedWatch/SafetyInformation/ucm458053.htm. Updated August 14, 2015. Accessed April 7, 2016.

10. Allergan Announces FDA Approval of ACZONE® (dapsone) Gel, 7.5\% for Treatment of Acne Vulgaris. Allergan. http://www.allergan.com/news/news/thomsonreuters/allergan-announces-fda-approval-of-aczone-dapsone. February 25, 2016. Accessed April 18, 2016.

11. LABEL: Aczone (dapsone) Gel, 7.5\%- dapsone gel. U.S. National Library of Medicine. https://dailymed.nlm.nih.gov/dailymed/drugInfo.cfm?setid=e3426f2f4d2b-40b4-ac26-e7d873c30f78. Accessed April 18, 2016.

12. Koo EB, Petersen TD, Kimball AB. Meta-analysis comparing efficacy of antibiotics versus oral contraceptives in acne vulgaris. J Am Acad Dermatol. 2014;71(3):450459.

13. Plovanich M, Weng QY, Mostaghimi A. Low usefulness of potassium monitoring among healthy young women taking spironolactone for acne. JAMA Dermatol.
2015;151(9):941-944

14. Cao H, Yang G, Wang Y, et al. Complementary therapies for acne vulgaris. Cochrane Database Syst Rev. 2015;1:CD009436.

15. Zheng W, Wu Y, Xu X, Gao X, Chen HD, Li Y. Evidence-based review of photodynamic therapy in the treatment of acne. Eur J Dermatol. 2014;24(4):444-456.

16. Liu Y, Zeng W, Hu D, et al. The long-term effect of $1550 \mathrm{~nm}$ erbium:glass fractional laser in acne vulgaris. Lasers Med Sci. 2016;31(3):453-457.

17. Paithankar DY, Sakamoto FH, Farinelli WA, et al. Acne treatment based on selective photothermolysis of sebaceous follicles with topically delivered light-absorbing gold microparticles. J Invest Dermatol. 2015;135(7):1727-1734.

18. Aslam I, Fleischer A, Feldman S. Emerging drugs for the treatment of acne. Expert Opin Emerg Drugs. 2015;20(1):91-101.

19. Trifu V, Tiplica E, Naumescu L, et al. Cortexolone 17 \-propionate cream, a new potent antiandrogen for the topical treatment of acne vulgaris. A pilot randomized, double-blind comparative study vs. placebo and tretinoin $0.05 \%$ cream. $\mathrm{Br} J \mathrm{Der}$ matol. 2011 165(1):177-183.

20. Qin M, Landriscina A, Rosen JM, et al. Nitric oxide-releasing nanoparticles prevent Propionibacterium acnes-induced inflammation by both clearing the organism and inhibiting microbial stimulation of the innate immune response. J Invest Dermatol. 2015;135(11):2723-2731

21. Hartman-Adams H, Banvard C, Juckett G. Impetigo: diagnosis and treatment. Am Fam Physician. 2014;90(4):229-235.

22. A Stronger Competitive Edge. FMX 101 for Moderate-to-Severe Acne. Foamix Pharmaceuticals. http://www.foamix.co.il/lead.asp?nodeID=549. Accessed March 2016.

23. Valente Duarte de Sousa IC. Novel pharmacological approaches for the treatment of acne vulgaris. Expert Opin Investig Drugs. 2014;23(10):1389-1410.

24. Mimetica MTC-896. AVARX. http://www.avarx.com/search/ showOpportunityDetails?asset_id=2460. Accessed March 2016.

25. Wilkin J, Dahl M, Detmar M, et al. Standard classification of rosacea: Report of the National Rosacea Society Expert Committee on the Classification and Staging of Rosacea. J Am Acad Dermatol. 2002;46(4):584-587.

26. Wollina U. Recent advances in the understanding and management of rosacea. F1000Prime Rep. 2014;6:50.

27. Del Rosso JQ, Thiboutot D, Gallo R, et al; American Acne \& Rosacea Society. Consensus recommendations from the American Acne \& Rosacea Society on the management of rosacea, part 5: a guide on the management of rosacea. Cutis. 2014;93(3):134-138.

28. van Zuuren EJ, Fedorowicz Z. Interventions for rosacea: abridged updated Cochrane systematic review including GRADE assessments. Br J Dermatol. 2015;173(3):651662

29. Piwnica D, Rosignoli C, de Ménonville ST, et al. Vasoconstriction and anti-inflammatory properties of the selective alpha-adrenergic receptor agonist brimonidine. $J$ Dermatol Sci. 2014;75(1):49-54.

30. Moore A, Kempers S, Murakawa G, et al. Long-term safety and efficacy of oncedaily topical brimonidine tartrate gel $0.5 \%$ for the treatment of moderate to severe facial erythema of rosacea: results of a 1-year open-label study. J Drugs Dermatol. 2014;13(1):56-61.

31. Highlights of prescribing information. MIRVASO. http://www.accessdata.fda.gov/ drugsatfda_docs/label/2013/204708lbl.pdf. Accessed March 2016.

32. Ilkovitch D, Pomerantz RG. Brimonidine effective but may lead to significant rebound erythema. J Am Acad Dermatol. 2014;70(5):e109-e110. doi: 10.1016/j. jaad.2014.01.853.

33. Routt ET, Levitt JO. Rebound erythema and burning sensation from a new topical brimonidine tartrate gel 0.33\%. J Am Acad Dermatol. 2014;70(2):e37-e38. doi: 10.1016/j.jaad.2013.10.054

34. Tanghetti EA, Jackson JM, Belasco KT, et al. Optimizing the use of topical brimonidine in rosacea management: panel recommendations. J Drugs Dermatol. 2015;14(1):33-40.

35. Stein L, Kircik L, Fowler J, et al. Efficacy and safety of ivermectin $1 \%$ cream in treatment of papulopustular rosacea: results of two randomized, double-blind, vehicle-controlled pivotal studies. J Drugs Dermatol. 2014;13(3):316-323.

36. Taieb A, Ortonne JP, Ruzicka T, et al; Ivermectine Phase III study group. Superiority of ivermectin $1 \%$ cream over metronidazole $0.75 \%$ cream in treating inflammatory lesions of rosacea: a randomized, investigator-blinded trial. $\mathrm{Br} J$ Dermatol. 2015;172(4):1103-1110.

37. Sobolewska B, Doycheva D, Deuter C, Pfeffer I, Schaller M, Zierhut M. Treatment of ocular rosacea with once-daily low-dose doxycycline. Cornea. 2014;33(3):257260 .

38. Schechter BA, Katz RS, Friedman LS. Efficacy of topical cyclosporine for the treatment of ocular rosacea. Adv Ther. 2009;26(6):651-659. 